\title{
FIVE-HUNDRED-METER APERTURE SPHERICAL TELESCOPE
} PROJECT

\author{
B. PENG, R. NAN, Y. SU, Y. QIU, L. ZHU and W. ZHU \\ Beijing Astronomical Observatory, National Astronomical Observatories, CAS, P.R. China \\ E-mail: pb@bao.ac.cn
}

\begin{abstract}
A Five hundred meter Aperture Spherical Telescope (FAST) is proposed to be built in the unique karst area of southwest China, and will act, in a sense, as a prototype for the Square Kilometer Array (SKA). It will be over twice as large as the Arecibo telescope coupled with much wider sky coverage. Some results from site surveys for such a SKA concept are briefly reported. Technically, FAST is not simply a copy of the existing Arecibo telescope but has rather a number of innovations. Firstly, the proposed main spherical reflector, by conforming to a paraboloid of revolution in real time through actuated active control, enables the realization of both wide bandwidth and full polarization capability while using standard feed design. Secondly, a feed support system which integrates optical, mechanical and electronic technologies will effectively reduce the cost of the support structure and control system. Pre-research on FAST has become a key project in the CAS.
\end{abstract}

Keywords: Spherical telescopc, karst depression, active reflector, cable supporting

Abbreviations: CAS - Chinese Academy of Sciences

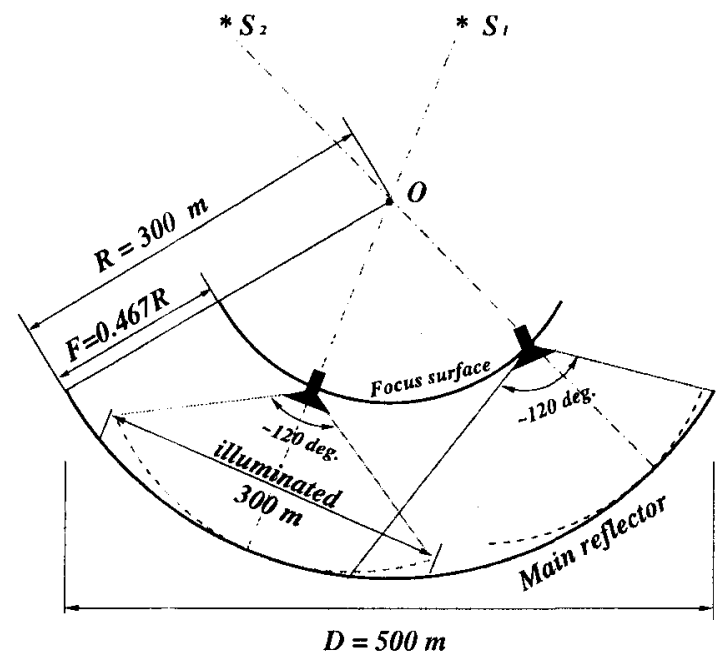

Figure 1. FAST concept and its geometrical configuration. 


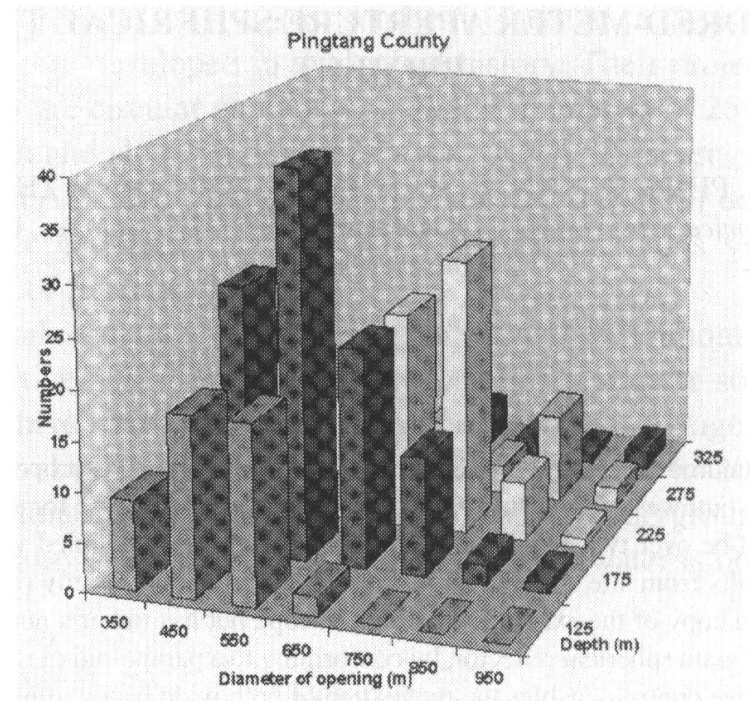

Figure 2. Statistics of Pingtang county depression geometry.

\section{Introduction}

FAST is to be constructed as a National Megascience Project of China, with an estimated cost of $\simeq 60$ M US\$ around the year 2005. Some basic parameters of FAST are, a main spherical reflector radius of $300 \mathrm{~m}$, a total of up to $500 \mathrm{~m}$ projected diameter, and an effective aperture of $300 \mathrm{~m}$ (Figure 1). The geometrical configuration will enable FAST to have a larger sky coverage ( $>40^{\circ}$ zenith angle) than the Arecibo telescope, and the simplified feed system will continuously cover the frequency range between 200 and $2000 \mathrm{MHz}$, with possible capability up to 5 or even $8 \mathrm{GHz}$ depending upon the cost. FAST will achieve the largest collecting area in the world.

\section{Site Survey and Interference Monitoring}

A large number of karst depressions in Guizhou province, at least 400 depressions, were investigated with Remote Sensing (RS), the Geographical Information System (GIS) and on-the-spot observations, and selected as candidate site locations. As an example, Figure 2 shows statistical results for Pingtang county. More than 10 depressions were imaged at the high resolution of $5 \mathrm{~m} / \mathrm{pixel}$, showing suitable profiles for a large spherical reflector (Peng et al., 1997).

In the selected sites, carbonate rock is the main hydrous layer. Wind speed measurements made near the ground at various heights above a depression were started in January, 1999. The speed grows from $1 \mathrm{~m} / \mathrm{s}$ in the bottom of the depressions to 7 
$\mathrm{m} / \mathrm{s}$ at the top. There are less than 5 days of snowfall per year, and no ice build-up at the sites.

A series of measurements at various sites has been carried out to check on their suitability, from the point of view of interference, for realizing FAST. The first measurements were made at 8 karst depression sites in November 1994 in both Pingtang and Puding counties. Further measurements were made in March 1995 for a period of a month in an attempt to understand distance effects. In June 2000, we re-monitored half of the above sites to see the change of interference with time. The results of these measurements provide information about the frequency, strength and characteristics of the interfering signals. Most of the interfering signals found appear to be narrow-band $(<10 \mathrm{kHz})$ beacon signals of unknown origin. Due to the remoteness of this region and local terrain shielding of karst hills, preliminary results of radio interference monitoring are quite promising (Peng et al., 1996).

\section{Active Main Spherical Reflector}

FAST can be seen as an 'Arecibo-type with active main reflector'. The telescope is 'pointed' by moving the feed cabin. It is well-known that the central part of a spherical surface deviates little from a paraboloid of revolution as a proper focal length is chosen, based on which, a novel design for a giant spherical reflector is proposed (Qiu, 1998). The illuminated part of the main spherical reflector is to be continuously adjustable to fit a paraboloid of revolution in real time by actuated active control, synchronous with the motion of the feed while tracking an object. A traditional feed system may then be adopted to achieve a broad bandwidth and full polarization capability through its freedom from spherical aberrations. If the focal length is set to be $0.467 \mathrm{R}(\sim 140 \mathrm{~m})$ in the real configuration shown in Figure 1, the range of travel required for the actuators is under $65 \mathrm{~cm}$ across the $300 \mathrm{~m}$ illuminated aperture. For the maximum apparent motion of the celestial objects, the rate of variation is found to be also very small, lower than $5 \mathrm{~cm} \cdot \mathrm{min}^{-1}$, which enables inexpensive solutions of the mechanical control.

For construction, it is necessary to divide the giant main spherical surface into smaller elementary units. Each element is a small part of the spherical surface and its curvature should be optimized to get the best fit to the paraboloid. Figure 3 shows one of the segmentation methods proposed (Su et al., 1999, Zheng, 1999). Firstly, the segmentation is done in a flat plane divided into $\sim 1800$ identical hexagons with sides $\sim 7.5 \mathrm{~m}$ long. Secondly, drop the plane into the spherical cap, keeping the length along the radial direction unchanged, while the dimension of the hexagon along the azimuth directon is shortened by a factor of sinc $(\theta)$, i.e., $L_{c}=L^{*} \operatorname{sinc}(\theta)$ (see Figure 3 ).

Each element has three actuators to fix its position and connect it with adjacent elements, and there would be an average of one actuator per element. If the r.m.s. of the aperture is expected to be smaller than $\lambda / 16(\sim 4 \mathrm{~mm})$ at $5 \mathrm{GHz}$, the largest 

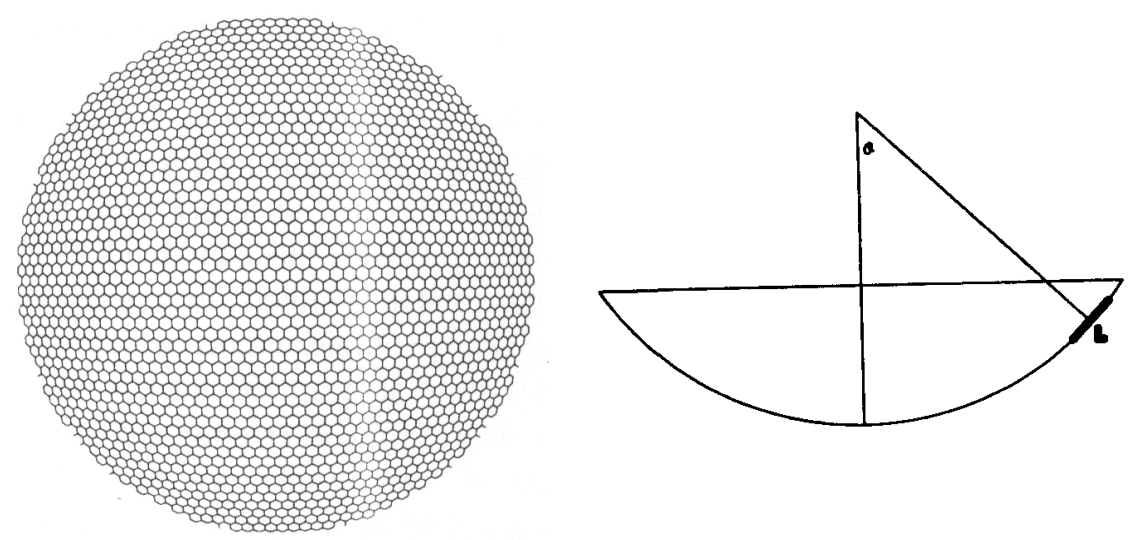

Figure 3. The FAST main reflector is segmented into about 1800 hexagons.

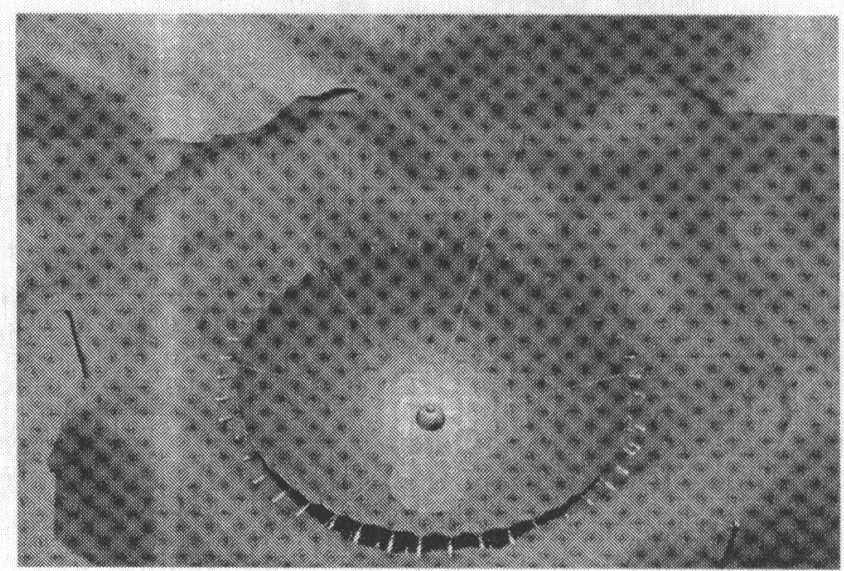

Figure 4. Cable support system without a platform.

dimension of each element in Figure 3 should not exceed $15 \mathrm{~m}$. Two possible means of supporting the surface have been considered, steel cables and concrete pillars. The final choice will depend upon the valley shape.

\section{Feed Support System}

\subsection{RECEIVER CABLE SUPPORT SYSTEM WITHOUT A PLATFORM}

A new design for the feed-support system (Figure 4) for FAST has been proposed by using six suspended cables connected to mechanical servocontrol systems (Duan et al., 1996). Compared with the Arecibo telescope, the total weight of the feed supporting system could evidently be reduced by such a design, probably from 


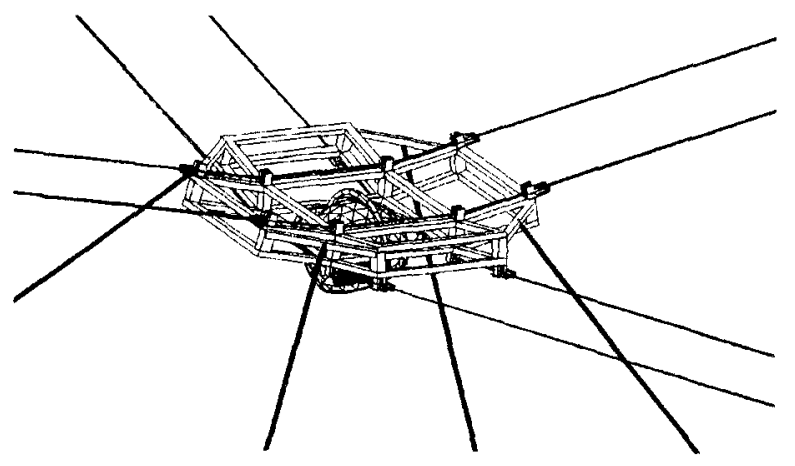

Figure 5. Cabin car driven by 2 pairs of parallel supporting and 4 downward cables.

nearly 10,000 to a few tens of tons. The tracking will be by means of integrated mechanical, electronic and optical technologies.

The whole system will mainly consist of three parts: firstly, the control system will consist of six sets of servo-mechanisms driven by a central computer. Given the difference between the apparent and required positions, where the feed (cabin) should point, the central computer will drive each servo-mechanism to adjust the position of the feed. Secondly, a group of receivers with a multi-beam feed will be mounted on a stabilizer in the focus cabin. This is to provide a second adjustment, since the cabin driven by cables alone may not achieve the pointing accuracy required. A laser ranging system (or CCD camera), being the third part, will be adopted to accurately measure the position of the feed in real time. The information will be fed back to the central computer for global loop control.

\subsection{Cable Car CONFiguration}

A small cable car, to serve as the focus cabin housing the feed and receivers, is to be driven by eight cables (Figure 5). Two pairs of parallel supporting cables will be suspended from four towers (instead of the three in the concept discussed above), while another four downward cables are securely fastened to four anchors which are symmetrically arranged about the main spherical reflector (Ren $e t$ al., 2001). The lengths of the connecting cables would be adjusted as the cabin location changes.

Positioning of the cabin would be achieved by driving the car on two crossed sets of supporting cables, which is like a trolley on the cableway in mountains. Rotation of the feed can be realized by a special mounting in the car. The axis of this mounting should intersect the center of gravity of the cabin. Actuators are to be employed for actively controlling any oscillations of the cabin induced by the motion.

The main advantages of this cable-car configuration are the following: firstly, the maximum length of cable extension will be relatively short, and the change may 
be as small as 20-30 $\mathrm{m}$ when observing a target; secondly, the downward cables (with a radius of $1 \mathrm{~cm}$ in such a design), can be used to adjust the stiffness and improve the dynamic characteristics of the system; thirdly, the car could be used as a crane during construction and maintenance of the main spherical reflector, and access for maintenance can be achieved by lowing the car down to a ground platform close to the tower foot.

\section{FAST Science}

The FAST will be especially effective in deep surveys for sources such as rare types of pulsars and neutral hydrogen clouds at moderately high redshifts. Details in the structure of individual pulses, or in their polarization, might be vital. Such measurements rely on raw sensitivity: a single pulse can only be observed once. FAST will give us the best information on the greatest number of pulsars. A $300 \mathrm{~m}$ telescope in combination with a $25 \mathrm{~m}$ one has the same response as two dishes of $87 \mathrm{~m}$. FAST as a VLBI station will be the hub of the most highly sensitive network. Studies of distant planets in the solar system are essential for understanding its evolution, the origins of life, and for investigating how the deep space environment would affect human beings with a view towards potential colonies in space. FAST will play an important role in the deep space network, and in SETI research.

\section{References}

Duan, B., Zhao, Y., Wang, J. and Xu, G.: 1996, Study of the feed system for a large radio telescope from the viewpoint of mechanical and structural engineering, in: Strom, R.G., Peng, B. and Nan, R. (eds.), Proc. of the $L T W G-3 \& W-S R T$, IAP, pp. 85-102.

Peng, B. and Nan, R.: 1997, Kilometer-square Area Radio Synthesis Telescope KARST project, IAU Symp. 179, 93-94.

Peng, B., Nan, R., Qiu, Y., Nie, Y., et al.: 1996, Further site survey for the next generation large radio telescope in Guizhou, in: Jackson, N. and Davis, R.J. (eds.), High Sensitivity Radio Astronomy. Cambridge Uni. Press, pp. 278-281.

Qiu, Y.: 1998, A novel design for a giant Arecibo-type Spherical radio telescope with an active main reflector, MNRAS 301, 827-830.

Ren, G., et al., 2001, ApSS, in press.

Su, Y., Zheng, Y., and Peng, B.: 1999, Schemes for segmenting the main reflector of the FAST, in: Proc. of the 4th EAMA, ASP, 94.

Zheng, Y.: 1999, Suggestions and comments on segmenting FAST reflector, MEMO to the FAST. 